\title{
Hammering Sound Frequency Analysis to Fix an Acetabular Cup during Total Hip Arthroplasty: Clinical Trials and Biomechanical Studies
}

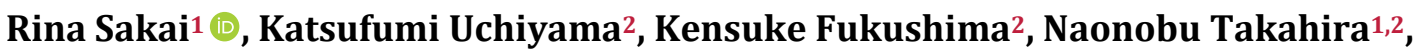 \\ Kazihiro Yoshida ${ }^{1}$, Masanobu Ujihira ${ }^{1}$
}

${ }^{1}$ Department of Biomedical Engineering and Technology, School of Allied Health Sciences, Kitasato University, Kanagawa, Japan; ${ }^{2}$ Department of Orthopedic Surgery, Kitasato University, Kanagawa, Japan

Correspondence to: Rina Sakai, rinax@kitasato-u.ac.jp

Keywords: Total Hip Arthroplasty, Hammering Sound, Frequency Analysis, Intraoperative Fracture, Loosening Received: December 26, $2020 \quad$ Accepted: January 23, $2021 \quad$ Published: January 26, 2021

Copyright $\odot 2021$ by author(s) and Scientific Research Publishing Inc.

This work is licensed under the Creative Commons Attribution International License (CC BY 4.0).

http://creativecommons.org/licenses/by/4.0/

\section{(c) (1) Open Access}

\section{ABSTRACT}

Failure during total hip arthroplasty may lead to bedridden of the elderly. Since the acetabulum cup fix in an anatomically deep region, failures, such as loosening and fracture, occur three times more frequently compared with failures of the stem fix in the femur. We investigated the possibility of evaluating whether fixation was acquired by frequency analysis of the hammering sound of implanting a cup into the acetabulum. The subjects were 11 patients (11 joints) who underwent total hip arthroplasty, biomechanical test materials, and orthopedic models. Surgeries and experiments were performed by orthopedists specialized in the hip. A system was constructed with a tablet PC and directional microphone, the peak frequency at which the amplitude reached the maximum was determined, and judgment processing (stable, unstable) of cup fixability was performed in real time. The stable maximum peak frequency observed in the clinical trials was $4.42 \pm 4.02 \mathrm{kHz}$. The mean stable maximum peak frequency in the biomechanical tests was $4.46 \pm 1.19 \mathrm{kHz}$ in biomechanical test materials and $4.56 \pm 2.02 \mathrm{kHz}$ in orthopaedicmodels. When hammering was continued, the frequency leading to fracture decreased in both biomechanical test materials and orthopaedicmodels. In conclusion, in clinical trials and biomechanical studies, variation of the maximum peak frequency decreased when fixation was acquired and the frequency stabilized. It was suggested that this method can serve as a fixability evaluation method of acetabular cups because analysis can be performed in real time during surgery, for which prevention of intraoperative fracture can be expected. 


\section{INTRODUCTION}

The total hip arthroplasty (THA) failure rate has been reported to be $4 \%$ - 30\% [1] [2] [3] [4]. THA failure is problematic because it extends the rehabilitation period of the patient and leads to bedridden of the elderly. Since the position of acetabular cup fixation in the acetabulum is located in an anatomically deep region, fixation failure occurs three times more frequently compared with those of stem set into the femur [5] [6] [7]. The cup can be fixed to the coxa by screws, but surgeons want to avoid using a screw as much as possible because the organs involved, which are present in the coxa, are life-threatening when they are damaged.

To prevent fixation failure, a hammering with strong impacting is employed to prevent loosening of the acetabulum and cup for a long time [8] [9]. However, blood vessels and nerves are present around the acetabulum and strong hammering has a risk of damaging the surrounding tissue. For this problem, there is no criterion to judge whether sufficient fixation was acquired at the time of cup placement and it depends on the surgeon's experience and sense [10].

The natural frequency of sound generated in an object is proportional to the Young's modulus and inversely proportional to the object's density and length. As a human pelvic and an artificial pelvic have different physical properties, the frequency characteristics of the hammering sounds produced from an artificial resin model will likely differ from those produced from the bone in a human pelvic.

Focusing on acoustic analysis in which pitch changes due to fixability of the subject, we have tried prediction of fracture during surgery by frequency analysis of the hammering sound of fixing a hip stem [11]. It was reported that the same peak frequency repeats when appropriate fixation is acquired during surgery, suggesting that intraoperative fracture can be prevented by stopping hammering at the time the peak frequency converged [12]. In this study, applying this technique to fixing a cup into the acetabulum by hammering, we investigated the possibility of predicting the occurrence of fracture during surgery in biological bone similarly to that in a bone model.

\section{MATERIALS AND METHODS}

\subsection{Objective and Materials}

This study was approved by IRB of the institution to which I belong (B11-93, B16-239). The subjects were 11 patients (11 joints) ( 4 male and 7 female patients, mean age: 71.6 years old (54 - 81 years old)) who underwent total hip arthroplasty at Kitasato University Hospital between October 2012 and November 2017. For the acetabular cup, G7 OsseoTiSysyrm (ZimmerBiomet) was used in all cases. The cup size was decided by templating by orthopedists specialized in the hip.

For the simulated bone, 16 each of a biomechanical test material (SKU1522-04, Sawbones) and an orthopedic model, Hemi pelvis with 54-mm acetabulum (SKU 1305, Sawbones), were used (Figure 1). The following abbreviations were used below: Patient's pelvis, PP; Biomechanical test material, BM; Orthopedic model, OM. G7 OsseoTiSysyrm (ZimmerBiomet) $54 \mathrm{~mm}$ was also used for the acetabular cup in the biomechanical studies.

\subsection{Measurement}

Surgeries and experiments were performed by orthopedists specialized in the hip joint with 20 years or longer clinical experience. After acetabuloplasty, the cup was set and hammering was applied to the acetabulum.

The system was constructed with a tablet PC (Miix2 8, Lenovo) and directional microphone (Mic FP-5500, SONY, Japan). Hammering sounds were collected at a sampling frequency of $44.1 \mathrm{kHz}$ and quantization bit rate of 16 bits and input into the tablet PC. Hammering sounds were subjected to shorttime Fourier analysis. The power spectrum was detected and the peak frequency at which the amplitude reached the maximum per hammering (maximum peak frequency) was determined and presented as a 

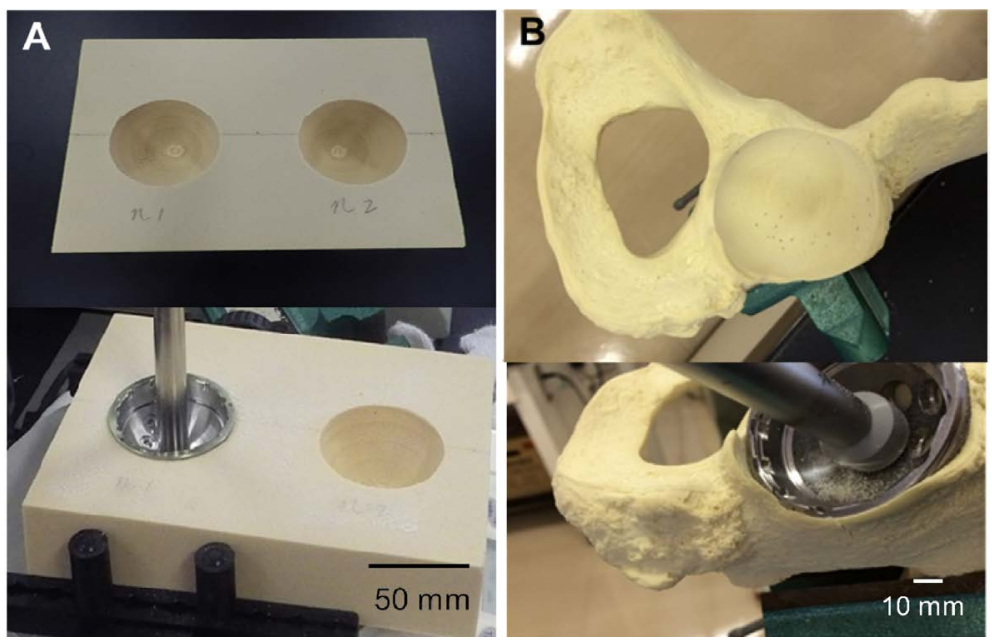

Figure 1. Top is materials and model. Bottom is fixation of an acetabular cup. (A) Biomechanical test materials (BM). (B) Hemi pelvis with $54 \mathrm{~mm}$ acetabulum of orthopaedic model (OM).

trend graph plotting the maximum peak frequency on the vertical axis and hammering count on the horizontal axis.

Real-time processing of judging whether cup fixation was acquired was performed. In the judgment, changes in the maximum peak frequency by $\pm 0.5 \mathrm{kHz}$ or larger were defined as unstable and not being fixed. When the maximum peak frequency changed within $\pm 0.5 \mathrm{kHz}$ or smaller in three consecutive judgments, the condition was defined as stable and judged as that the cup was fixed.

\section{RESULTS}

In the clinical trials, the maximum peak frequency judged as stable was present in all PP. The mean stable maximum peak frequency was $4.42 \pm 4.02 \mathrm{kHz}$ (Table 1). A constant maximum peak frequency continued $3.27 \pm 0.47$ times. No failure occurred during or after THA in any patient. There were no cases of intraoperative or postoperative failures in the diagnosis by the surgeon.

In $\mathrm{BM}$, the stable maximum peak frequency was present in all 16 cases (Table 2) and the stable maximum peak frequency was $4.46 \pm 1.19 \mathrm{kHz}$. Cracks and fractures were noted in 9 of the 16 cases of the biomechanical test material. The maximum peak frequency at which a crack or fracture was produced was $1.28 \pm 1.12 \mathrm{kHz}$, clarifying that it was lower than the frequency regarded as fixation.

In $\mathrm{OM}$, the maximum peak frequency repeated high and low in the trend graph before reaching fixation, as shown in Figure 2(A). A stable maximum peak frequency was present in 13 of the 16 model cases and the maximum peak frequency was $4.56 \pm 2.02 \mathrm{kHz}$. In the trend graphs in which the cup was judged as fixed, the maximum peak frequency was judged as equivalent 3 times or more, as shown in Figure 2(B). A value with variation within $\pm 0.5 \mathrm{kHz}$ was regarded as equivalent. A crack or fracture was noted in all OM cases. When hammering was continued and caused fracture, the frequency decreased in both OM and BM (Figure 3).

\section{DISCUSSION}

It has been reported that the initial fixability of the acetabular cup can be evaluated based on the waveform detected by a load sensor attached to an impact hammer [13]. Slipping of the sensor into patient's body is of concern for this method, but the frequency can be non-invasively analyzed, being advantageous [14]. 
Table 1. Stabled maximum peak frequencies and counts of consecutive equivalent peak frequencies of patient pelvic.

\begin{tabular}{cc}
\hline Stabled frequency $(\mathrm{kHz})$ & Counts of consecutive equivalent peak frequencies (times) \\
\hline 0.60 & 3 \\
2.00 & 3 \\
3.60 & 3 \\
3.86 & 3 \\
3.88 & 3 \\
3.90 & 3 \\
5.10 & 3 \\
5.63 & 4 \\
5.90 & 4 \\
6.30 & 3 \\
7.88 & $3.27 \pm 0.47$ \\
$4.42 \pm 4.02$ & \\
\hline
\end{tabular}

Table 2. Stable and fractured maximum peak frequencies using biomechanical test materials and orthopedic models.

\begin{tabular}{cccc}
\hline & BM & \multicolumn{2}{c}{ OM } \\
\hline $\begin{array}{c}\text { Stabled frequency } \\
(\mathrm{kHz})\end{array}$ & Fractured frequency & Stabled frequency & Fractured frequency \\
$(\mathrm{kHz})$ & No fracture & 1.22 & 0.73 \\
3.6 & 0.52 & 3.66 & 0.80 \\
3.7 & No fracture & 3.76 & 0.80 \\
3.9 & No fracture & 4.09 & 0.58 \\
3.9 & 0.59 & 5.28 & 0.78 \\
3.9 & 0.57 & 5.33 & 0.56 \\
3.9 & No fracture & 5.33 & 0.72 \\
4.3 & 0.52 & 5.33 & 0.57 \\
4.3 & 2.99 & 5.33 & 0.88 \\
4.4 & 0.53 & 5.33 & 0.57 \\
4.5 & 3.41 & 5.53 & 0.88 \\
4.5 & No fracture & 5.53 & 0.50 \\
4.6 & 1.18 & 5.53 & 0.60 \\
4.8 & 0.56 & unstable & 0.71 \\
5.5 & No fracture & unstable & 0.70 \\
7.8 & No fracture & unstable & 0.70 \\
$4.46 \pm 1.19$ & $1.28 \pm 1.12$ & $4.56 \pm 2.02$ & $0.71 \pm 0.11$ \\
\hline & & & \\
\hline & & & \\
\hline
\end{tabular}



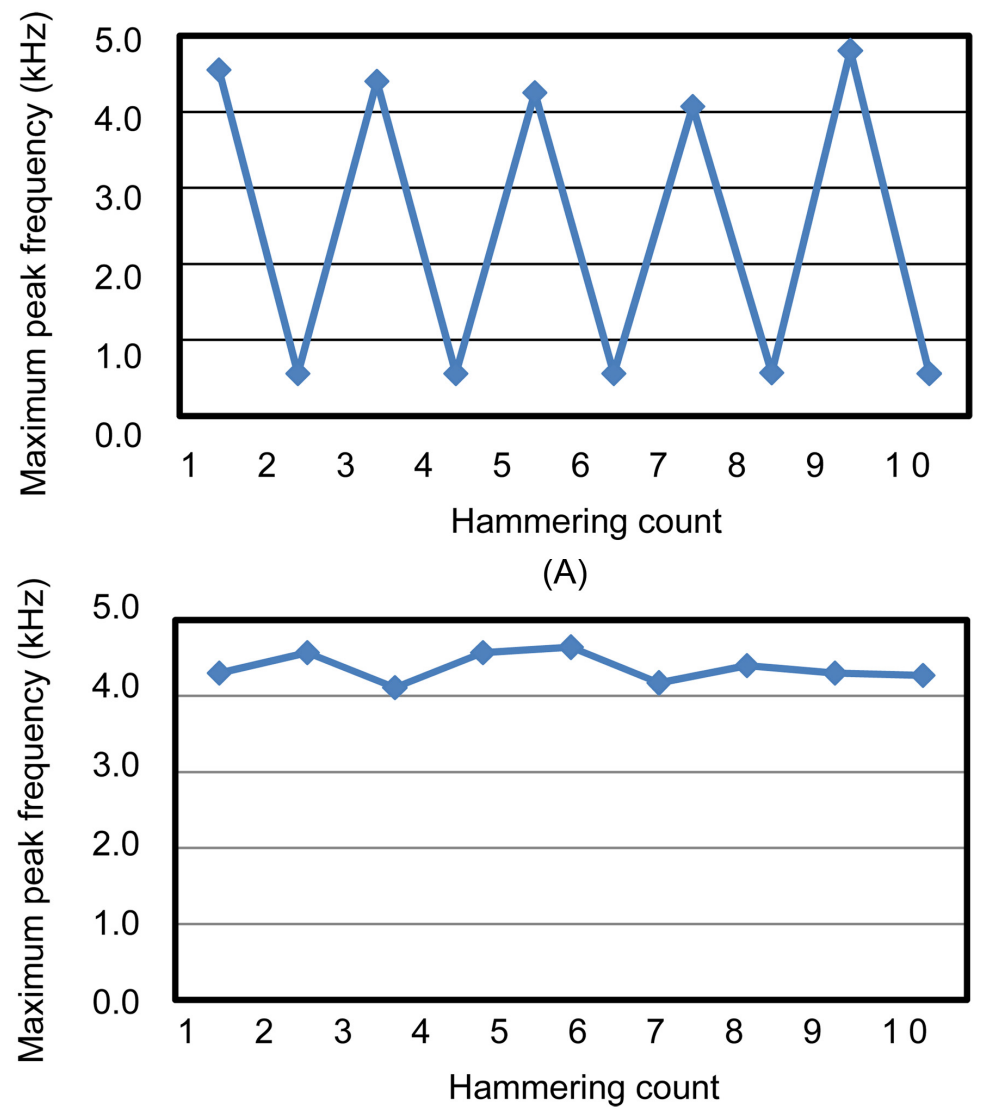

(B)

Figure 2. Trend graph of maximum peak frequency at a hammering count before fixation. (A) Unstable. (B) Stable.

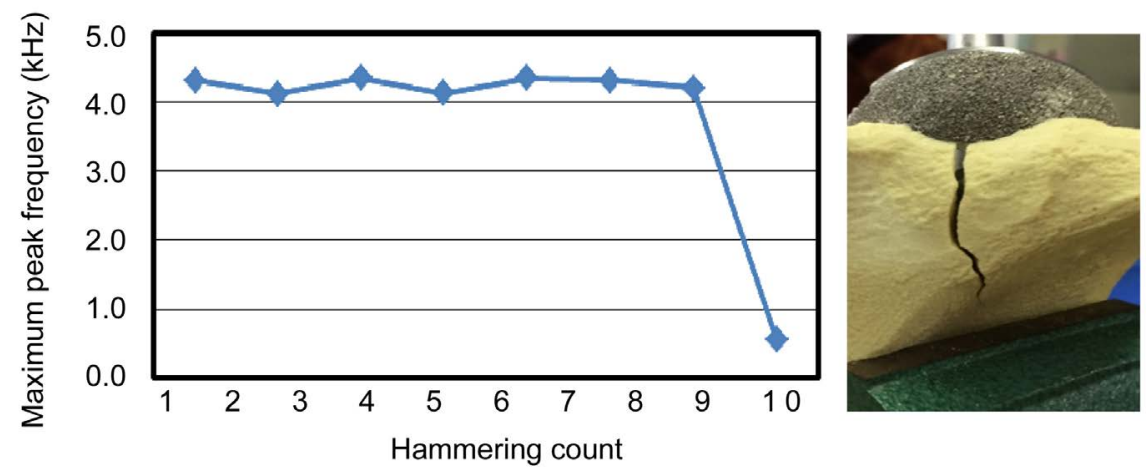

Figure 3. Left is a trend graph of maximum peak frequency at a hammering count. Right is a fracture of the orthopedic model that occurred on the 10th time.

It has been reported that the same frequency continues when an artificial joint acquires fixability [15]. In our study, "stable" was defined as "maximum peak frequency $\pm 0.5 \mathrm{kHz}$ ". A constant maximum peak frequency was noted at a high rate in all model cases, suggesting that the maximum peak frequency is useful to alert to stop hammering. For clinical application, the accuracy is low at $\pm 0.5 \mathrm{kHz}$. It is necessary to consider the interval. 
A system giving an alarm when a stable maximum peak frequency continues should be prepared. The safety factor $S$ of medical devices is 2 [16]. Considering that the counts of consecutive equivalent peak frequencies of PP was 3.27, a count of 3 was estimated to be appropriate to start the alarm.

Repeating high and low maximum peak frequencies before stabilization may have been due to instability of the cup unevenly contacting the acetabulum. In a case showing instability, there may have been a margin to further continue hammering. The standard deviation of PP depends on the variability of the patient's bone quality. In BM, the standard deviation of the maximum peak frequency decreased as expected because the density and length were constant. The reason for not reaching fracture in many cases was the block shape with thickness around the cup. Since the size and density were uniformed in OM, it is valid that the standard deviation of the maximum peak frequency decreased compared to that in PP. Fracture occurred in all OM cases because anatomically, there is a thin bone region, but the fracture site varied, such as the bottom of the acetabulum and region hit by the rim of the cup.

It has been clarified that when a mild fracture (including cracks) occurred, the frequency decreased by $3 \mathrm{kHz}$ [17]. In the present study, when a crack or fracture was produced in the coxa, the frequency decreased. This is related to the fact that the natural frequency of an object is inversely proportional to the vibration length and we considered that it was due to relative extension of the length of the vibrating region by a crack produced in the acetabulum.

\section{CONCLUSION}

In conclusion, in clinical trials and biomechanical studies, variation of the maximum peak frequency decreased when fixation was acquired and the frequency stabilized. It was suggested that this method can serve as a fixability evaluation method of acetabular cups because analysis can be performed in real time during surgery, for which prevention of intraoperative fracture can be expected.

\section{ACKNOWLEDGEMENTS}

The authors disclosed receipt of the following financial support for the research, authorship, and/or publication of this article: This work was supported by the KAKENHI Grant Number JP 26820160 and 18K04179. We are grateful to Yasuo Otsu, Masahiro Kakeshita, and Takanori Usui for their support.

\section{CONFLICTS OF INTEREST}

The authors declare no conflicts of interest regarding the publication of this paper.

\section{REFERENCES}

1. Foster, A.P., Thompson, N.W., John, W. and Charlwood, A.P. (2005) Periprosthetic Femoral Fractures-A Comparison between Cemented and Uncemented Hemiarthroplasties. Injury, 36, 424-429. https://doi.org/10.1016/j.injury.2004.07.023

2. Lindahl, H. (2007) Epidemiology of Periprosthetic Femur Fracture around a Total Hip Arthroplasty. Injury, 38, 651-654. https://doi.org/10.1016/j.injury.2007.02.048

3. Moroni, A., Faldini, C., Piras, F. and Giannini, S. (2000) Risk Factors for Intraoperative Femoral Fractures during Total Hip Replacement. Annales Chirurgiae et Gynaecologiae, 89, 113-118.

4. Schwartz, J.T., Mayer, J.G. and Engh, C.A. (1989) Femoral Fracture during Non-Cemented Total Hip Arthroplasty. Journal of Bone and Joint Surgery American Volume, 71, 1135-1142. https://doi.org/10.2106/00004623-198971080-00003

5. Callaghan, J.J., Templeton, J.E., Liu, S.S., Pedersen, D.R., Goetz, D.D., Sullivan, P.M. and Johnston, R.C. (2004) Results of Charnley Total Hip Arthroplasty at a Minimum of Thirty Years. A Concise Follow-Up of a Previous Report. Journal of Bone and Joint Surgery American Volume, 86, 690-695. 
https://doi.org/10.2106/00004623-200404000-00004

6. Digas, G., Kärrholm, J. and Thanner, J. (2006) Different Loss of BMD Using Uncemented Press-Fit and Whole Polyethylene Cups Fixed with Cement: Repeated DXA Studies in 96 Hip Randomized to 3 Types of Fixation. Acta Orthopaedica, 77, 218-226. https://doi.org/10.1080/17453670610045948

7. Mathieu, V., Michel, A. and Lachaniette, C.H.F. (2013) Variation of the Impact Duration during the in Vitro Insertion of Acetabular Cup Implants. Medical Engineering \& Physics, 35, 1558-1563. https://doi.org/10.1016/j.medengphy.2013.04.005

8. Albrektsson, T., Brånemark, P.I., Hansson, H.A. and Lindström, J. (1981) Osseointegrated Titanium Implants: Requirements for Ensuring a Long-Lasting, Direct Bone-to-Implant Anchorage in Man. Acta Orthopaedica Scandinavica, 52, 155-170. https://doi.org/10.3109/17453678108991776

9. Kroeber, M., Ries, M.D., Suzuki, Y. and Renowitzky, G. (2002) Impact Biomechanics and Pelvic Deformation during Insertion of Press-Fit Acetabular Cups. The Journal of Arthroplasty, 17, 349-354. https://doi.org/10.1054/arth.2002.30412

10. Sakai, R., Takahashi, A., Takahira, N., Uchiyama, K., Yamamoto, T., Uchida, K., Fukusima, K., Moriya, M., Takaso, M., Itoman, M. and Mabuchi, K. (2011) Hammering Force during Cementless Total Hip Arthroplasty and Risk of Microfracture. Hip International, 21, 330-335. https://doi.org/10.5301/hip.2011.8408

11. Sakai, R., Kikuchi, A., Morita, T., Takahira, N., Uchiyama, K., Yamamoto, T., Moriya, M., Uchida, K., Fukusima, K., Tanaka, K., Takaso, M., Itoman, M. and Mabuchi, K. (2011) Hammering Sound Frequency Analysis and Prevention of Intraoperative Periprosthetic Fractures during Total Hip Arthroplasty. Hip International, 21, 718-723. https://doi.org/10.5301/HIP.2011.8823

12. Sakai, R., Uchiyama, K., Takhira, M., Kakeshita, M., Otsu, Y., Yoshida, K. and Ujihira, M. (2020) Usefulness of Hammering Sound Frequency Analysis as an Evaluation Method for the Prevention of Trouble during Hip Replacement. Journal of Biomedical Science and Engineering, 13, 74-80. https://doi.org/10.4236/jbise.2020.135007

13. Michela, A., Boscb, A., Sailhanc, F., Vayrona, R. and Haiat, G. (2016) Ex Vivo Estimation of Cementless Acetabular Cup Stability Using an Impact Hammer. Medical Engineering \& Physics, 38, 80-86. https://doi.org/10.1016/j.medengphy.2015.10.006

14. Whitwell, G., Brockett, C.L., Young, S., Stone, M. and Stewart, T.D. (2013) Spectral Analysis of the Sound Produced during Femoral Broaching and Implant Insertion in Uncemented Total Hip Arthroplasty. Proceedings of the Institution of Mechanical Engineers, Part H: Journal of Engineering in Medicine, 227, 175-180. https://doi.org/10.1177/0954411912462813

15. Jaecques, S.V., Pastrav, C., Zahariuc, A. and Van der Perre, G. (2004) Analysis of the Fixation Quality of Cementless Hip Prostheses Using a Vibrational Technique. Proceedings of ISMA, Leuven, 20-22 September 2004, 443-456. https://doi.org/10.1115/ESDA2004-58581

16. Hwang, T.J., Sokolov, E., Franklin, J.M. and Kesselheim, A.S. (2016) Comparison of Rates of Safety Issues and Reporting of Trial Outcomes for Medical Devices Approved in the European Union and United States: Cohort Study. BMJ, 353, i3323. https://doi.org/10.1136/bmj.i3323

17. Sakai, R., Yamamoto, T., Uchiyama, K., Takahira, N., Kakeshita, M, Otsu, Y., Yoshida, K. and Ujihira, M. (2020) Prediction of Intraoperative Fracture by Hammering Sound Frequency Analysis and Stress Estimation during Total Hip Arthroplasty. Journal of Biomedical Science and Engineering, 13, 113-119. https://doi.org/10.4236/jbise.2020.136011 